\title{
A Novel Low Power Binary to Gray Code Converter Using Gate Diffusion Input (GDI)
}

\author{
${ }^{1}$ Pakkiraiah chakali, ${ }^{2} \mathrm{~K}$ Sreekanth Yadav, ${ }^{3}$ Dilli Babu S \\ ${ }^{I}$ ECE Department, Sree Vidyanikethan Engineering College (Autonomous), JNTUA, Anantapur Tirupati, \\ INDIA, \\ ${ }^{2}$ ECE Department, Sree Vidyanikethan Engineering College (Autonomous), JNTUA, Anantapur. Tirupati, \\ INDIA, \\ ${ }^{3}$ ECE Department, Sree Vidyanikethan Engineering College (Autonomous), JNTUA, Anantapur, Tirupati,
} INDIA.

\begin{abstract}
In modern era, Ultra low power design has an Active research topic due to its various Applications. In this paper we introduce a novel low power and Area efficient Binary to Gray code converter is implemented by using four transistor XOR gate. This four Transistor XOR gate is designed by using Gate Diffusion Input(GDI).Both four transistor and Binary to Gray code converter is designed and implemented by using Mentor Graphics Tool. So we were obtained the power dissipation of Binary to Gray code conveter which is very small.
\end{abstract}

Keywords: - Low Power, OR, Binary code, Gray code, Area, GDI Technique

\section{INTRODUCTION}

The design of code converters[14],[16] which forms the basic building blocks of all digital VLSI circuits[9] has been undergoing a considerable improvement, being motivated by basic design goals, viz. minimizing the transistor count, minimizing the power consumption[5].The XOR[1] gates form the fundamental building block of code converters. Enhancing the performance of the XOR gates can significantly improve the performance of the code converters.

Different types of XOR gates that have been realized over the years.

The code converters are more complex and power consuming circuits in digital design. To reduce the power dissipation several code converters are designed but they are not suitable for operation in the subthreshold region. These design requires more transistors leads to area is increasing, so these are not suitable for small and low price systems. The power consumption techniques are CMOS complementary logic, Pseudo nMOS, Dynamic CMOS[7], Clocked CMOS logic $\left(\mathrm{C}^{2} \mathrm{MOS}\right)$, CMOS Domino logic, Cascade voltage switch logic (CVSL)[12], Modified Domino logic, Pass Transistor Logic (PTL)[8].The most useful low power consumption

technique is PTL. The PTL advantages are,

1) High speed, due to small node capacitances.

2) Low power dissipation[4], as a result of reduced number of transistors.

3) Lower interconnection effect, due to smaller area. There are two main drawbacks in PTL,

1) The threshold voltage across the single channel pass transistors results in reduced drive and hence slower

operation at reduced voltages.

2)The high input voltage level is not VDD the PMOS device in inverter is not fully turned off. In order to overcome these drawbacks we use Transmission Gate(TG)[9] logic.

The main advantage of the TG logic is complex logic functions are implemented by using small number of transistors. Another advantage is logic level swing can be reduced by using TG. The combination of NMOS PT with CMOS output inverters is called Complementary pass transistor logic (CPL).It suffers from the static power and low swing at gates of the output inverters. To reduce the static power dissipation and full swing operation we use the Double pass transistor logic (DPL). Double pass transistor logic (DPL) [3] has more area due to presence of PMOS transistor.

\section{PRINCIPLE OF GDI TECHNIQUE}

A new technique solves most of the problems like low power and less area known as Gate-DiffusionInput (GDI) [2] is proposed. This technique is used to reducing power consumption, propagation delay, and area of digital circuits. The GDI method is based on the simple cell shown in Figure.1. A basic GDI cell contain s four terminals $-\mathrm{G}$ (common gate input of nMOS and pMOS transistors), $\mathrm{P}$ (the outer diffusion node of pMOS 
transistor), $\mathrm{N}$ (the outer diffusion node of nMOS transistor), and D (common diffusion node of both transistors) [10].

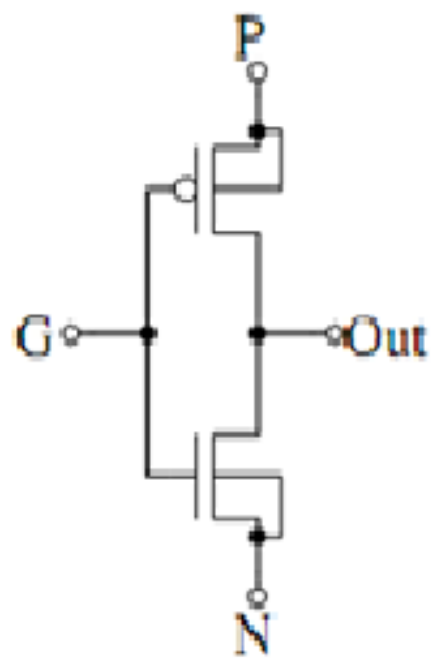

Fig.1. Basic Gate-Diffusion-Input Cell.

TABLE 1

Truth Table of the Basic GDI Cell

\begin{tabular}{|c|c|c|c|c|}
\hline N & P & G & OUT & Function \\
\hline 0 & B & A & $\bar{A} B$ & F1 \\
\hline B & 1 & A & $\bar{A}+\mathrm{B}$ & OR \\
\hline 1 & B & A & A+B & AND \\
\hline B & 0 & A & AB & MUX \\
\hline C & B & A & A + AC & NOT \\
\hline 0 & 1 & & $\bar{A}$ & \\
\hline
\end{tabular}

\section{IMPLEMENTATION OF EX-OR}

The basic building gate of Binary to Gray code converter is EX-OR. The implementation of EX-OR gate using Gate-Diffusion-Input (GDI)[10] is shown in figure2.The design and implementation of EX-OR gate using Gate-Diffusion-Input (GDI) is dissipates less power and it requires less are.

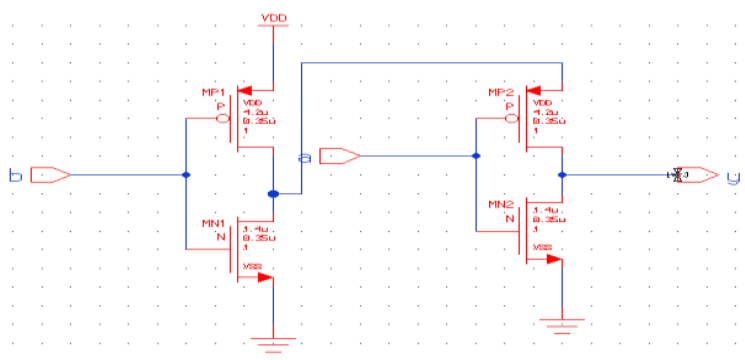

Fig. 2. XOR cell with the GDI technique

\section{IMPLEMENTATION OF GRAY TO BINARY CODE}

A binary code [13] is a way of representing text or computer processor instructions by the use of the binary number system's two-binary digits 0 and 1 . This is accomplished by assigning a bit string to each particular symbol or instruction. For example, a binary string of eight binary digits (bits) can represent any of 256 possible values and can therefore correspond to a variety of different symbols, letters or instructions. In 
computing and telecommunication, binary codes are used for any of a variety of methods of encoding data, such as character strings, into bit strings. Those methods may be fixed-width or variable-width. In a fixed-width binary code, each letter, digit, or other character, is represented by a bit string of the same length; that bit string, interpreted as a binary number[15], is usually displayed in code tables in octal, decimal or hexadecimal notation.

The reflected binary code, also known as Gray code. It is a binary numeral system where two successive values differ in only one bit. It is a non-weighted code. The reflected binary code was originally designed to prevent spurious output from electromechanical switches. Today, Gray codes [18] are widely used to facilitate error correction in digital communications such as digital terrestrial television and some cable TV systems. Patent applications give "Gray code" as an alternative name for the "reflected binary code". one of those also lists "minimum error code" and "cyclic permutation code" among the names. The problem with natural binary codes is that, with real (mechanical) switches, it is very unlikely that switches will change states exactly in synchrony. In the transition between the two states shown above, all three switches change state. In the brief period while all are changing, the switches will read some spurious position. Even without key bounce, the transition might look like $011-001-101-100$. When the switches appear to be in position 001, the observer cannot tell if that is the "real" position 001, or a transitional state between two other positions. If the output feeds into a sequential system (possibly via combinational logic) then the sequential system may store a false value. The reflected binary code solves this problem by changing only one switch at a time, so there is never any ambiguity of position.

\begin{tabular}{|c|c|c|}
\hline $\begin{array}{c}\text { Decimal } \\
\text { number }\end{array}$ & Gray code & $\begin{array}{c}\text { Binary } \\
\text { number }\end{array}$ \\
\hline 0 & 000 & 000 \\
\hline 1 & 001 & 001 \\
\hline 2 & 011 & 010 \\
\hline 3 & 010 & 011 \\
\hline 4 & 110 & 100 \\
\hline 5 & 111 & 101 \\
\hline 6 & 101 & 110 \\
\hline 7 & 100 & 111 \\
\hline
\end{tabular}

More formally, a Gray code is a code assigning to each of a contiguous set of integers, or to each member of a circular list, a word of symbols such that each two adjacent code words differ by one symbol. These codes are also known as single-distance codes, reflecting the Hamming distance of 1 between adjacent codes. There can be more than one Gray code[16] for a given word length, but the term was first applied to a particular binary code for the non- negative integers, the binary-reflected Gray code, or BRGC, the three-bit version of which is shown above.
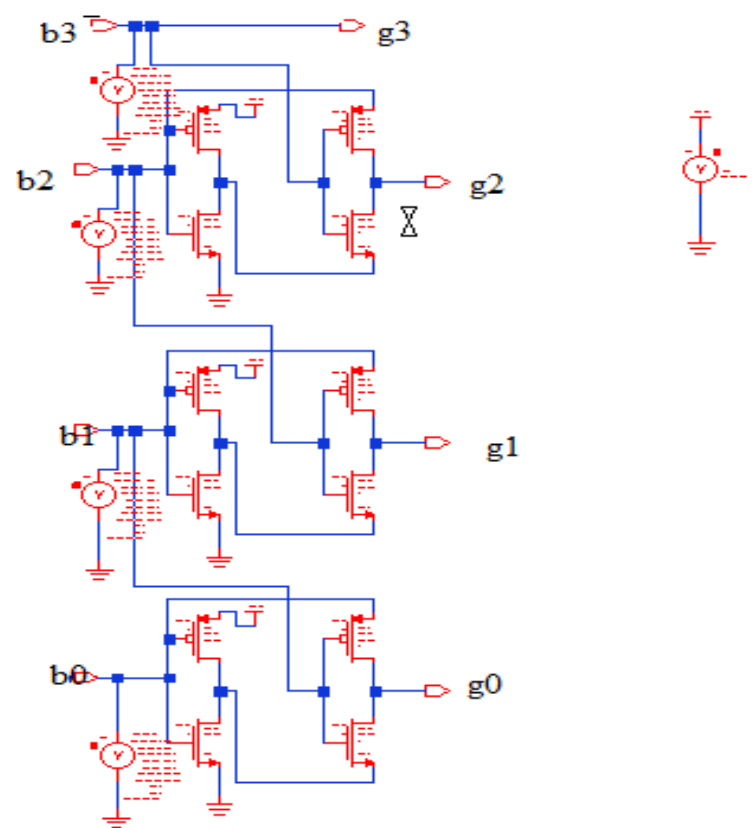

Fig. 3. Binary to Gray Code with the GDI technique 


\section{RESULTS AND DISCUSSION}

The Binary to Gray code converter operates in $66 \mathrm{MHz}$ range. In Mentor Graphics Tool both EX-OR and Binary to Gray code converter are designed. After that we were simulated this designs. By using simulation results we got the values of rise time, fall time, delay, power dissipation and we were taken the input and output simulated waveforms. The simulated waveforms are shown in figure4-5.The power dissipation and transistor count are shown in table2.

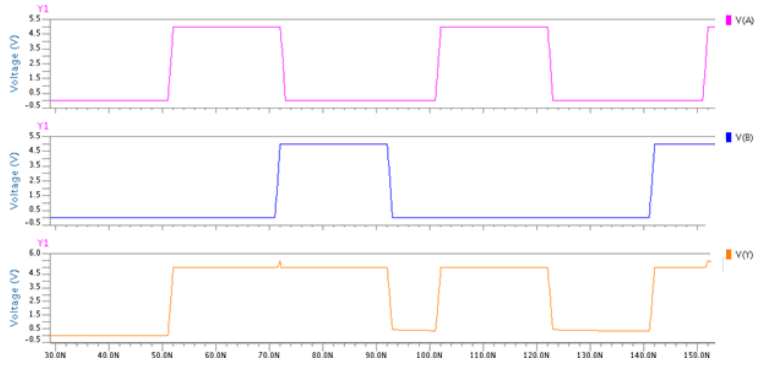

Fig.4.waveforms at $5 \mathrm{v}$ and $66 \mathrm{MHZ}$ of $\mathrm{XOR}$

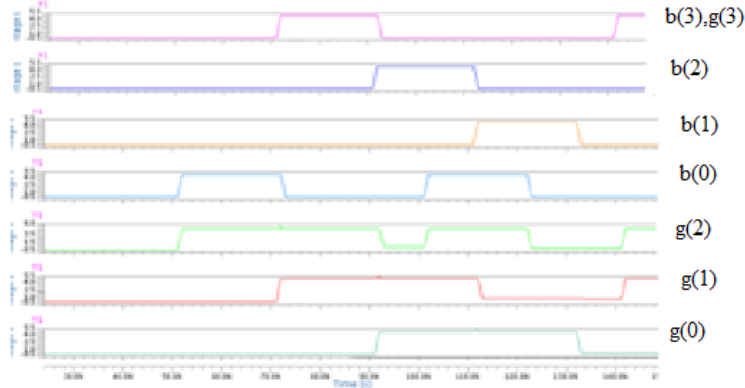

Fig.5.waveforms at 5v and 66MHZ of Binary to Gray Code.

Table.2 comparison of Code Converters (Power, Number of Transistors).

\begin{tabular}{|c|c|c|}
\hline Circuit & $\begin{array}{c}\text { No } \\
\text { Transistors }\end{array}$ & $\begin{array}{c}\text { Power } \\
\text { (w) }\end{array}$ \\
\hline Binary to Gray & 12 & $461.1958 \mathrm{P}$ \\
\hline EX-OR & 4 & $126.31 \mathrm{P}$ \\
\hline
\end{tabular}

\section{CONCLUSION}

A new technique, Gate-Diffusion-Input (GDI) technique has been adopted for reducing the transistor count with full swing. The GDI technique has been implemented in Code Converters and the comparison results have been shown. The performance metrics like area, power, delay and transistor count are compared with the previous CMOS logic design families. The implementation of Binary to Gray Code Converter has been presented in GDI technique and can be extended to Other codes. The future research activities may include integration of the proposed Binary to Gray Code in complex digital systems, digital communication systems and telecommunications.

\section{REFERENCES}

[1] Pakkiraiah Chakali, Design of High Speed Six Transistor Full Adder using a Novel Two Transistor XOR Gates,IJARCSEE ISSN: $2277-9043$.

[2] Pakkiraiah Chakali, A Novel Low power and Area efficient Carry Look Ahead Adder Using GDI Technique, IJRCET

[3] Jan M. Rabaey, Anantha Chandrakasan and Borivoje Nikolic, "Digital Integrated Circuits- A Design Perspective",2 nd ed., Prentice Hall of India Pvt Ltd, New Delhi,2006.

[4] A. P. Chandrakasan, S. Sheng, and R. W. Brodersen, "Low-power CMOS digital design". IEEE J. Solid-State Circuits, vol. 27, pp. 473-484, Apr. 1992.W.-K. Chen, Linear Networks and Systems (Book style). Belmont, CA: Wadsworth, 1993, pp. 123-135.

[5] A. P. Chandrakasan and R.W. Brodersen, "Minimizing power consumption in digital CMOS circuits". Proc. IEEE, vol. 83, pp. 498523, Apr. 1995

[6] H. P. Alstead and S. Aunet, "Seven subthreshold flip-flops cells," in Proc. IEEE Nor CHIP 2007, Nov. 2007, pp. 1-4.

[7] N. Weste and K. Eshraghian Principles of CMOS digital design. 
[8] W. Al-Assadi, A. P. Jayasumana, and Y. K. Malaiya, "Pass-transistor logic design,” Int. J. Electron., vol. 70, pp. 739-749, 1991.

[9] I. S. Abu-Khater, A. Bellaouar, and M. I. Elmastry, "Circuit techniques for CMOS low-power high-performance multipliers," IEEE J. Solid-State Circuits, vol. 31, pp. 1535-1546, Oct. 1996.

[10] A. Morgenshtein, A. Fish, I.A. Wagner, "Gate-Diffusion Input (GDI) - A Power Efficient Method for Digital Combinational Circuits," IEEE Trans. VLSI, vol.10, no.5 pp.566-581, October 2002.

[11] A. Morgenstein, A. Fish, I. Wagner, “An Efficient Implementation of D-Flip-Flop Using the GDI Technique," ISCAS “04, pp. 673676, May 2004.

[12] M. Morris Mano and Michael D.Ciletti, "Digital Design".

[13] Table of general binary codes. An updated version of the tables of bounds for small general binary codes given in M.R. Best, A.E. Brouwer, F.J. Mac Williams, A.M. Odlyzko \& N.J.A. Sloane (1978), "Bounds for Binary Codes of Length Less than 25", IEEE Trans. Inf. Th. 24: 81-93.

[14] Table of Nonlinear Binary Codes. Maintained by Simon Litsyn, E. M. Rains, and N. J. A. Sloane.

[15] Glaser, Anton (1971). "Chapter VII Applications to Computers". History of Binary and other No decimal Numeration. Tomash. ISBN 0-938228-00-5. cites some pre-ENIAC milestones.

[16] Black, Paul E. Gray code. 25 February 2004. NIST.

[17] Press, WH; Teukolsky, SA; Vetterling, WT; Flannery, BP (2007). "Section 22.3. Gray Codes". Numerical Recipes: The Art of Scientific Computing (3rd ed.). New York: Cambridge University Press. ISBN 978-0-521-88068-8.

[18] Savage, Carla (1997). "A Survey of Combinatorial Gray Codes". SIAM Rev. 39 (4):605629doi:10.1137/S0036144595295272. JSTOR 2132693.

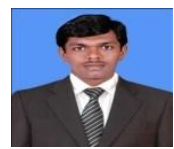

C.Pakkiraiah completed his B.Tech in Electronics and Communication Engineering from Sreenivasa Institute of Technology and management studies, Chittoor, Andhra Pradesh, India in 2009. He is now pursuing his Master of Technology (M.Tech) in VLSI at Sree Vidyanikethan Engineering College, Tirupati, Andhra Pradesh, India. His interest includes Digital Design, ASIC Design, and VLSI Testing.

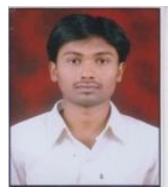

K Sreekanth Yadav completed his B.Tech in Electronics and Communication Engineering from Mekapati Rajamohan Reddy Institute Of Technology and Science, Udayagiri, Nellore, Andhra Pradesh, India in 2011. he is now pursuing his Master of Technology (M.Tech) in VLSI at Sree Vidyanikethan Engineering College, Tirupati, Andhra Pradesh, India. His interest includes Digital Design, FPGA Design

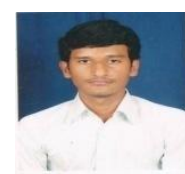

S Dilli Babu completed his B.Tech in

Electronics and Communication Engineering from Kuppam Engineering College, Kuppam, Chittoor, Andhra Pradesh, India in 2011. he is now pursuing his Master of Technology (M.Tech) in VLSI at Sree Vidyanikethan Engineering College, Tirupati, Andhra Pradesh, India. His interest includes Digital Design, ASIC Design and FPGA Design. 